\title{
FUNDAMENTOS DEL LÁSER Y SU APLICACIÓN EN UROLOGÍA
}

\author{
David Vázquez Alba y Joaquín Carballido Rodríguez.
}

Servicio de Urología. Hospital Universitario Puerta de Hierro-Majadahonda. Universidad Autónoma de Madrid. Madrid. España.

\begin{abstract}
Resumen.- El láser, dispositivo de amplificación de luz por emisión estimulada de radiación, se trata de un dispositivo capaz de transformar otras energías en radiación electromagnética emitiendo haces de luz de distintas longitudes de onda. Se trata de aparatos que amplifican la luz y producen haces de luz coherentes cuya frecuencia va desde el infrarrojo hasta los rayos X. La emisión estimulada, proceso en que se basa el Láser, fue descrita por A. Einstein en 1917, pero no es hasta la década de los 60, cuando se observó el primer proceso láser en un cristal de rubí. Según el medio que emplean, los láseres suelen denominarse de estado sólido, de gas, semiconductores o líquidos.
\end{abstract}

Los posibles usos del láser son casi ilimitados, convirtiéndose en una herramienta muy valiosa dentro de las Ciencias biomedicas, gracias a los diversos efectos (fotovaporización, foto- disrupción, fotocoagulación o fotoestimulación) que provoca al interactuar con los tejidos. Por este motivo, hoy día, el uso de láseres en el campo de la Urología nos ofrece un amplio abanico de posibilidades, que van desde la cirugía desobstructiva como la fragmentación de un cálculo o la resección y ablación del tejido prostático hasta la cirugía reconstructiva como es la soldadura de tejidos en la vasovasostomía o la reparación de una estenosis uretral.

Palabras clave: Fotoquímica. Fotocoagulación. Fotovaporización. Fotodisrupción. Láser.

Summary.- A laser, light amplification by stimulated emission of radiation, is a device able to transform other energies into electromagnetic radiation with emission of light beams of different wavelengths. They amplify the light and produce coherent light beams, the frequency of which varies from infrared to $X$ ray. Stimulated emission, the process laser is based on, was described by A. Einstein in 1917, but it was not until the decade of the '60s when the first laser process was observed in a ruby crystal. Depending on the environment they use, lasers may be named as solid-state, gas, semiconductors or liquid.

The possibility of uses for laser is almost unlimited, becoming a very valuable tool in biomedical sciences thanks to the various effects they produce when interacting with tissues /photovaporization, photodisruption, photocoagulation or photostimulation). For this reason, today, the use of lasers in the field of urology offers a wide range of possibilities, going from surgery for the treatment of obstruction, such as the fragmentation of a urinary stone or resection/ablation of prostatic tissue, to reconstructive surgery, such as tissue welding in vasovasostomy or urethral stenosis repair.

Keywords: Photochemistry. Photocoagulation. Photovaporization. Photodisruption. Laser. 


\section{INTRODUCCIÓN}

La tecnología láser es una de las áreas del moderno desarrollo tecnológico que mayor desarrollo ha tenido en los últimos 50 años. En líneas generales, podemos afirmar que el láser se trata de un dispositivo capaz de transformar otras energías (eléctrica, química, electromagnéti$c a$, etc.), en radiación electromagnética emitiendo haces de luz de distintas longitudes de onda. De esta emisión de luz es de donde el láser toma su nombre: Light Amplification by Stimulated Emision of Radiation (1).

Hasta finales del siglo XIX, los estudios sobre la luz se limitaban a analizar su comportamiento. Así, los científicos eran capaces de identificar las fuentes productoras de luz, pero al mismo tiempo, eran incapaces de conocer los mecanismos por la que estas fuentes la generaban. En 1870, Maxwel, desarrolló la teoría de las ondas electromagnéticas en que las propiedades físicas de la luz encuentran un marco coherente. Según esta teoría, la radiación electromagnética es una modalidad de propagación de energía sin el soporte del medio material. En este sentido, la radiación electromagnética puede considerarse como una doble onda, formada por dos campos, eléctricos y magnético, que se encuentran en fase y cuyos planos de propagación son perpendiculares. El conjunto de todas las radiaciones electromagnéticas conocidas se pueden ordenar de mayor a menor frecuencia, en función de menor a mayor longitud de onda. Esta ordenación constituye lo que se conoce con el nombre de espectro de radiación electromagnética.

El carácter ondulatorio de la radiación electromagnética explico una serie de fenómenos físicos, pero la descripción puramente ondulatoria, resultaba insuficiente para explicar otros fenómenos tales como la interacción de la radiación con la materia, o la absorción y emisión de energía por parte de los átomos. Tales fenómenos fueron correctamente interpretados a principios del siglo XX por físicos como Planck y Einstein. Describiendo que cuándo las ondas electromagnéticas interaccionan con la materia, se produce un intercambio de energía a través de pequeños paquetes, denominados cuantos de radiación o fotones $(2,3)$.

En 1900, Planck, estudiando las radicaciones del "cuerpo negro" introdujo el "cuanto de energía" como una realidad física, al subdividir el continuo de energía en elementos de tamaño finito, asignándoles un valor constante y proporcional a su frecuencia $(2,3)$.

En 1917, Einstein, enuncia los principios de la teoría de emisión espontánea (4). Así, el primer principio afirma que la luz viaja en paquetes de energía denominados fotones. El fotón puede considerarse como un "corpúsculo" de energía sin soporte material, esta energía es múltiplo de la frecuencia de la onda electromagnética asociada y viene dada por la ecuación $\mathbf{E}=\mathbf{h v}$ (donde $\mathbf{h}$ es una constante física, Ilamada constante de Planck y $\mathbf{v}$ es la frecuencia). Por tanto, una radiación electromagnética de frecuencia " $v$ " no puede adquirir ni ceder energía más que en cantidades discretas del valor hv, lo que nos permite interpretar la radiación electromagnética desde el punto de vista corpuscular.
El segundo principio de Einstein, implica que la mayoría de átomos y moléculas existentes en la naturaleza se encuentran en un estado de baja energía $E_{0}$. Así, que un átomo, en determinadas condiciones, y tras ser estimulado por la luz o cualquier otra fuente energética, queda en estado de excitación, pasando a un nivel de energía superior. Tras la excitación, el átomo tiende a volver a su estado inicial de baja energía, liberando espontáneamente energía en forma de fotones.

Einstein también descubrió, que las partículas cargadas pierden su energía al interaccionar con la materia, existiendo tres tipos de interacciones fundamentales (2):

- Colisión elástica: la partícula choca con los átomos del medio desviándose de su trayectoria y cediendo una cierta cantidad de energía.

- Colisión inelástica: la partícula interacciona con los electrones atómicos transfiriendo a estas pequeñas cantidades de energía y provocando que el electrón atómico escape a la atracción del núcleo (ionización del átomo) o que el electrón pase a un estado menos ligado (excitación del áto$\mathrm{mo})$.

- Colisión radiactiva: la partícula cargada de "frena" o se "desvía" en su interacción con los átomos del medio y como resultado emite ondas electromagnéticas (radiación de frenado).

En 1958, Townes y Shawlow en EEUU, simultáneamente a Basov y Projorov en la URSS, elaboraron el primer modelo teórico del láser, al formular las condiciones necesarias para amplificar la emisión estimulada de ondas. Trabajo que les valió el Premio Nóbel de Física en 1964 (5).

Cuarenta y tres años después que Einstein enunciara sus primeras teorías, Maiman introduce el primer láser de rubí para uso comercial y su primera aplicación en el campo de la Medicina, será la extirpación de un tumor en la retina (6) (Figura 1).

Los pioneros en aplicar el láser en Urología fueron Parsons en 1968 en Carcinoma de pene (7), Mulvaney y Beck en 1968 en la litofragmentación de cálculos vesicales con láser rubí (8) y Watson y Dretler en 1984 con láser con colorante pulsado (verde cumarina) en la litofragmentación de litiasis ureterales (9).

\section{¿CÓMO FUNCIONA EL LÁSER?}

Según la teoría cuántica y basándonos en el modelo atómico de Bohr, enunciado en 1913, donde los electrones giran alrededor del núcleo en orbitales energéticos claramente definidos, y siendo el átomo incapaz de emitir radicaciones de forma continua, ya que los electrones permanecen alrededor del núcleo en orbitas estacionarias. Únicamente cuando los electrones cambian de orbita, se produce la emisión de energía. Podemos afirmar que cualquier elemento químico presenta una serie de niveles de energía de valor prefijado; en estos niveles se sitúan los electrones cuya carga es negativa mientras que en el nú- 
cleo atómico, formado por neutrones y protones presenta una carga positiva. Se establece así, que los electrones sólo pueden girar en ciertas órbitas de radios determinados. Estas órbitas son estacionarias, y en ellas el electrón no emite energía: la energía cinética del electrón equilibra exactamente la atracción electrostática entre las cargas opuestas de núcleo (+) y electrón (-).

El electrón solo puede tomar así los valores de energía correspondientes a esas órbitas. Los saltos de los electrones desde niveles de mayor energía a otros de menor energía o viceversa suponen, respectivamente, una emisión o una absorción de energía electromagnética en forma de paquetes o quantum de energía (fotones de luz) $(2,3)$.

La forma más estable del átomo se da cuando tiene sus electrones situados en los niveles de energía más bajos posibles. Por lo tanto, cuando hay un electrón con un nivel de energía dado, en un átomo que presenta una o más plaza para electrones de menor energía, el electrón en cuestión tiende a ir a los niveles más bajos de energía liberando un fotón.

Se puede conseguir que una sustancia (generalmente una mezcla de sólidos o gases) que tengan un gran número de átomos excitados, libere un fotón de alguno de ellos; de esa manera se puede inducir en los átomos vecinos el mismo fenómeno (reacción en cadena) y la consecuente liberación de energía, aumentando el fenómeno en progresión geométrica hasta la obtención de un haz de fotones que conformará el láser (emisión estimulada de radiación).

Los láseres obligan a los átomos a almacenar luz y emitirla de forma coherente. Primero, los electrones de los átomos del láser son bombardeados hasta un estado de excitación por una fuente de energía. Después, se los estimula mediante fotones externos para que emitan

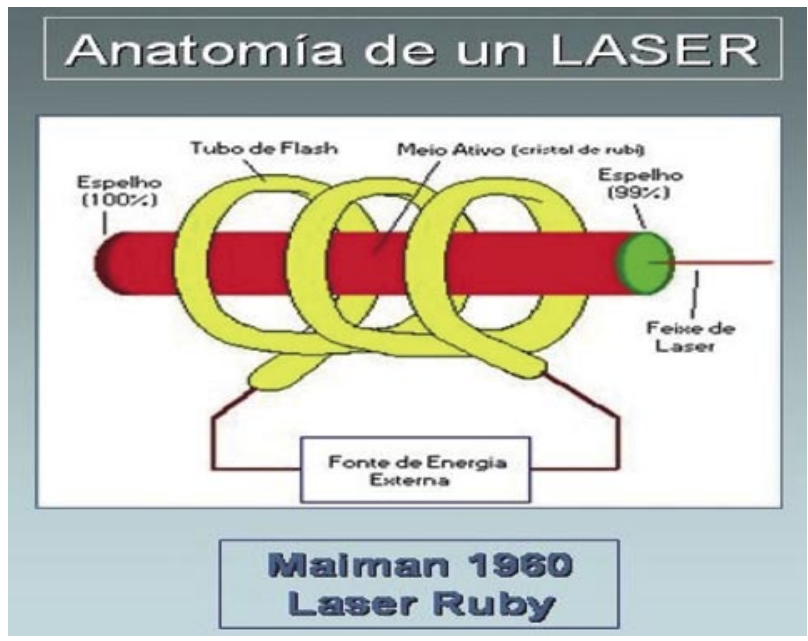

FIGURA 1. Anatomía láser rubí de Maiman (1960). la energía almacenada en forma de fotones, mediante un proceso conocido como emisión estimulada. Los fotones emitidos tienen una frecuencia que depende de los átomos en cuestión y se desplazan en fase con los fotones que los estimulan. Los fotones emitidos, a su vez, chocan con otros átomos excitados y liberan nuevos fotones. La luz se amplifica a medida que los fotones se desplazan hacia atrás y hacia delante entre dos espejos paralelos desencadenando nuevas emisiones estimuladas. Al mismo tiempo, la luz láser emitida, se filtra por uno de los espejos, que es solo parcialmente reflectante.

El láser, al igual que la luz, se refleja, se refracta y viajan a $3 \times 10^{8} \mathrm{~m} / \mathrm{s}$; pero a diferencia de ésta, sus haces son coherentes (todos los fotones emitidos están en la misma fase, lo que le confiere una gran potencia de acción), unidireccionales y monocromáticos (todos los fotones tienen la misma longitud de onda y frecuencia) (10) (Figura 2).

\section{TIPOS DE LÁSER}

Los láseres pueden clasificarse según los siguientes criterios: material de fabricación, potencia, tipo y frecuencia de emisión.

Conforme al material de fabricación, los láseres pueden ser de estado sólido, de gas, de semiconductores o líquidos:

1. Láseres de estado sólido: los medios más comunes en este tipo de láseres son varillas de cristal de rubí o vidrios y cristales con impureza de neodimio. Los láseres de estado sólido proporcionan las emisiones de mayor energía. Normalmente funcionan con pulsos, generando un destello de luz en un tiempo breve.

2. Láseres de gas: el medio de un láser de gas puede ser un gas puro, una mezcla de gases o incluso un vapor metáli$\mathrm{co}$, que suele estar contenido en un tubo cilíndrico de vidrio o cuarzo, con dos espejos situados en sus extremos. Entre los láseres de gas, destacan los de helio-neón por su elevada estabilidad de frecuencia, pureza de color y la mínima dispersión del haz. El láser de Argón similar al anterior y el láser de dióxido de carbono, formado por una mezcla de $\mathrm{CO}_{2}, \mathrm{~N}_{2}$ y Helio, que son láseres de onda continua, muy potentes, eficientes y utilizados en la industria.

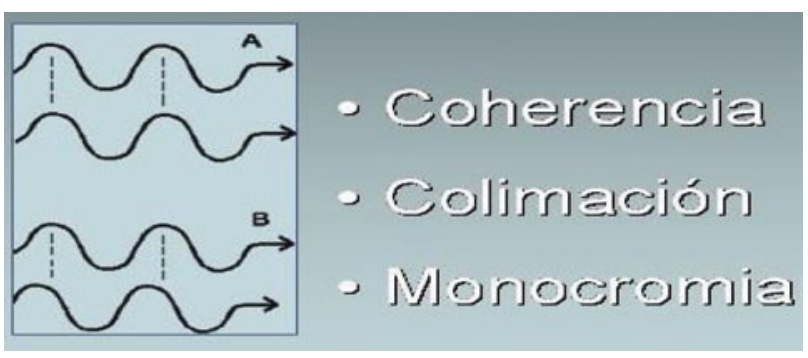


3. Láseres de semiconductores: son los láseres más compactos y están formados por una unión de varias capas de semiconductores con diferentes propiedades de conducción eléctrica. El arseniuro de galio es el semiconductor más utilizado. Entre los usos más comunes de este tipo de laser están los reproductores de discos compactos y las impresoras láser.

4. Láseres líquidos: los medios más comunes de este tipo de láseres son tintes inorgánicos (cumarina, rodamina b) contenidos en recipientes de vidrio (láser Dye).

5. Láseres de electrones libres: son láseres capaces de producir radiación de haces de electrones, no ligados a átomos, que circulan a lo largo de las líneas de un campo magnético.

En cuanto a la potencia, los láseres se dividen en los de baja potencia $(<1$ W) como el láser Helio-Neón o el semiconductor, y los de alta potencia (>1w) como el láser de $\mathrm{CO}_{2}$ y el de Neodimio.

En referencia al tipo y frecuencia de emisión de los distintos láseres, podemos distinguir láser de emisión pulsada o no pulsada, dependiendo de que la emisión sea continua o discontinua. La pulsación se realiza por medio de un oscilador y tiene como objeto ampliar la gama de aplicaciones y mejorar el control sobre algunos de los efectos biológicos. La frecuencia de emisión puede ir desde el infrarrojo hasta el ultravioleta pasando por una amplia de colores del espectro visible, en forma seriada o simultanea.

\section{MECANISMO DE ACCIÓN DEL LÁSER}

Cuando aplicamos un láser sobre un tejido orgánico, este interactúa con las células de dicho tejido, provocando los siguientes efectos 1 1-16:

- Fotodisrupción: dando lugar al estallido celular por la elevada temperatura (pasa de $37^{\circ}$ a $2000^{\circ}$ ) que adquiere la célula como consecuencia de la gran liberación de energía. Este efecto es utilizado para la destrucción de células malignas y se requiere láseres de gran potencia (Neodimio o de $\mathrm{CO}_{2}$ )

- Fotovaporización: se produce la evaporación del agua de la célula con la que interacciona el láser, pudiendo incrementar la temperatura tisular de $37^{\circ}$ a $400^{\circ}$ (láser de $\left.\mathrm{CO}_{2}\right)$.

- Fotocoagulación: la interacción del láser con la célula provoca cambios en la estructura terciaria y cuaternaria de las proteínas celulares, elevando la temperatura de $37^{\circ}$ a $65^{\circ}$ y provocando en consecuencia la fotocoagulación de la sangre (láser de Argón y láser Verde).

- Fotorradiacción: la interacción del láser con el tejido provoca una elevación muy leve de la temperatura, en 1-2 grados y este efecto de calor local, actuaría acelerando los procesos fisiológicos (láseres blandos o de baja potencia).
- Fotoestimulación o fotoquímica: el efecto más fascinante pero a la vez desconocido para el cual se han propuesto diversas hipótesis: existencia de un bioplasma como segundo cuerpo en todos los seres vivos, que tendría propiedades semiconductoras y sería el receptor de mensajes eutróficos. Otra hipótesis propone que el láser reduce la carga negativa en la membrana celular, alterando su permeabilidad y en consecuencia favoreciendo el intercambio de algunos iones. Por tanto tras la administración de una sustancia fotosensibilizadora, las células malignas pueden retener a su vez dichas sustancias favoreciendo la acción de los láseres que tienen afinidad (por ejemplo la porfirinas y protoporfirinas). El resultado es una reacción fotoquímica que liberaría radicales libres a nivel intracelular provocando la muerte celular. Este efecto ha sido utilizado la terapia fotodinámica de tumores de piel y para el diagnóstico del carcinoma in situ de vejiga (HEXVIX).

- Fotoacústico o mecánico: se basa en la conversión de la energía de diferentes tipos de láser en ondas de choque que fundamentan la litotricia

\section{LÁSERES USADOS EN UROLOGÍA}

Como hemos expuesto en apartados anteriores, existen en la actualidad diversos tipos de láseres en función de diferentes criterios, pero no todos tienen aplicabilidad en la práctica urológica habitual. Así, dentro de todos ellos podemos destacar (17-19):

- Láser Rubí: fue el primer láser producido a partir de un cristal de rubí sintético (6). Produce de forma pulsada un haz de luz roja con una longitud de onda de $694 \mathrm{~nm}$. Este tipo de láser se utilizado para eliminar lesiones cutáneas y tatuajes, pero en urología tiene escasa aplicabilidad, usándose exclusivamente en el tratamiento de lesiones cutáneas a nivel genital y para la eliminación del vello perineal en las uretroplastias.

- Láser $\mathrm{CO}_{2}$ : emite una luz con una longitud de onda de 10,600 nm. El haz de luz emitido es altamente absorbido por el agua, provocando la fotovaporización de los tejidos en un espesor de aproximadamente $1 \mathrm{~mm}$ y la fotocoagulación de los mismos, sobre los $0,5 \mathrm{~mm}$ de profundidad (20).

- Láser Neodimium: Yttrium-Aluminum-Garnet (Láser NdYAG): el ión $\mathrm{Nd}^{3+}$ puede estar presente en diferentes cristales, pero los más usados en la actualidad son los cristales de $\mathrm{Y}_{3} \mathrm{Al}_{5} \mathrm{O}_{12}$ por presentar una serie de características diferenciales, como son: mayor eficiencia, mayor calidad óptica, y una elevada conductividad térmica, lo que nos permite alta tasas de repetición. El láser Nd-YAG emite un haz de luz con una longitud de onda de $1064 \mathrm{~nm}$ (cerca del infrarrojo), lo cual permite una mayor penetración en los tejidos (más de $10 \mathrm{~mm}$ de profundidad), obteniendo una buena hemostasia (coagulando vasos de $5 \mathrm{~mm}$ de diámetro). Además, presenta propiedades de corte y favorece la litofragmentación intracorpórea. Estando indicado su uso en urología para la litofragmentación, fotocoagulación de tumores uroteliales y la incisión en estenosis urológicas (21-23). 
El láser Nd-YAG de doble frecuencia y doble pulso (FREDDY), se trata de un láser con pulso corto y de doble frecuencia, con una longitud de onda de 532 a 1064 $\mathrm{nn}$. Es por tanto, un laser de baja potencia y bajo coste desarrollado principalmente para la litotricia intracorpórea $(24,25)$.

- Láser Potassium-Titanyl Phosphate crystal (Láser KTP o láser verde): es un láser $\mathrm{Nd}-\mathrm{YAG}$ cuyo haz de luz pasa a través de un cristal de Potasio (K), Titanyl (T) y Fosfato $(\mathrm{P})$, emitiendo un haz de luz visible en la parte verde del espectro electromagnético y con una longitud de onda de onda de $532 \mathrm{~nm}$. Se trata de un láser, mal absorbido por el agua, siendo en cambio muy bien absorbido por la hemoglobina. Por lo cual, penetra mucho menos en el espesor del tejido. Su indicación en Urología es para realizar incisiones, resección y ablación del tejido prostático e incisiones en la patología estenótica urológica (26-29).

- Láser Dye: el haz de luz emitido por este tipo de láser presenta una longitud de onda variable en función del colorante o tinte usado. El colorante más comúnmente utilizado es la cumarina verde, el cual cuando es estimulado produce una luz con una longitud de onda de $504 \mathrm{~nm}$. Se emplea principalmente para la litotricia intracorpórea y la ablación de lesiones vasculares. A diferencia de los láseres sólidos, requiere el recambio del tinte como consecuencia del desgaste, siendo esto un inconveniente e incrementando el gasto derivado del mantenimiento del equipo, en relación con otros sistemas de láser (30).

- Láser Alexandrita: se trata de un láser compuesto por un mineral conocido como alexandrita $\left(\mathrm{BeAl}_{2} \mathrm{O}_{4}\right)$. El haz de luz emitido, se caracteriza por una longitud de onda comprendida entre el rango de 700-830 nm. Este haz de luz es absorbida por la melanina y puede ser usado para lesiones cutáneas. En urología se utiliza principalmente para la litotricia intracorpórea (3).

- Láser Diodo semiconductor: produce un haz de luz con una longitud de onda comprendida entre los 805-1000 $\mathrm{nm}$, en función de la adicción de elementos como aluminio o indio. Este tipo de láser es usado para la coagulación de tejidos y para el tratamiento térmico de lesiones sólidas, incluyendo la próstata. Así, la energía del láser es liberada en el interior del tejido mediante una fibra óptica, incrementando localmente la temperatura y provocando una desnaturalización de las proteínas tisulares (13,31-36).

- Láser Holmium: Ytrium Aluminum Garnet (Ho:YAG): se trata de un láser sólido compuesto por un elemento mineral denominado Holmiun, dispuesto en el interior de un cristal YAG, capaz de emitir un haz de luz con una longitud de onda de $2150 \mathrm{~nm}$. Se utiliza de forma pulsada y su mecanismo de acción es fototérmico. Es usado fundamentalmente en urología para la patología benigna de próstata (incisión, ablación, resección y enucleación de la glándula prostática), así como para la litogragmentación intracorpórea, donde constituye el "gold stardard" de la litotricia láser (al presentar un escaso efecto fotoacústico) por generar ondas de presión de baja amplitud lo que reduce el daño mecánico sobre uréter y riñón.
- Láser de Nitrógeno: este láser incorpora a el gas inerte $\mathrm{N}_{2}$ como medio del láser y cuando es excitado por energía, es capaz de provocar un haz de luz con una longitud de onda de $337 \mathrm{~nm}$. Este tipo de láser es usado en el diagnóstico de tumoraciones uroteliales mediante técnicas de autofluorescencia (37).

- Láser Erbium: Yttrium Aluminum Garnet (Er:YAG): este láser esta siendo estudiada su aplicabilidad en el terreno de la urología. Estudios sugieren que es muy superior al Ho: YAG para el tratamiento ablativo de la próstata, provocando mínimo daño térmico en las estructuras adyacentes y que parece tener una mayor eficacia para la fragmentación intracorpórea de litasis de oxalato cálcico. Además, el láser Er:YAG puede ser usado, con mayor precisión, que el láser Ho:YAG, para las incisiones de corte frío a nivel de la mucosa uretral y ureteral. El principal problema que plantea este tipo de láser es el elevado coste de la fibra óptica que se usa durante la intervención $(35,38-40)$.

- Láser Thulium: Yttrium Aluminum Garnet (Th:YAG): actualmente en estudio, pero reciente investigaciones ponen de manifiesto que podría ser competir a corto plazo con el láser $\mathrm{HO} Y \mathrm{YAG}$, al provocar un mínimo daño en los tejidos colaterales, Además, los resultados iniciales en litofragmentación son bastante esperanzadores (41-44).

Como resumen de los diferentes tipos de láser y sus distintas aplicaciones médicas, podemos agruparlos en función de sus diferentes utilidades en el campo de la urología:

- Para incisiones y corte fríos en tejidos: (uretra, ureter, endopielotomías, cuello vesical) disponemos del Ho:YAG, ND:YAG o el KTP.

- Para resección o ablación: (HPB, carcinoma de pene, condilomas genitales, tumores vesicales) podríamos utilizar el $\mathrm{Nd}: Y A G, \mathrm{Ho:YAG}, \mathrm{KTP}$, láser diodo o el láser $\mathrm{CO}_{2}$.

- Para la litotricia endocorpórea: (pelvis renal, uréter, vejiga) usaremos el Ho:YAG, FREDDY, láser diodo pulsado o el láser alexandrita.

- Para soldadura de tejidos: (vasovasostomía, reconstrucciones uretral, hipospadia, etc.) disponemos del láser diodo, $\mathrm{KTP}, \mathrm{Nd}: \mathrm{YAG}$ o láser $\mathrm{CO}_{2}$.

- Para la autoflurescencia: (diagnóstico de tumores uroteliales) usamos el láser de Nitrógeno.

- Para la eliminación de vello: (uretroplastias con parche de piel) disponemos del láser rubí, láser alexandrita, láser diodo o el Nd:YAG.

\section{BIBLIOGRAFÍA y LECTURAS RECOMENDADAS (*lectura de interés $y^{* *}$ lectura fundamental)}

1. COLLS, J.: "La terapia láser hoy". $3^{\mathrm{a}}$ Ed. Barcelona: Centro de Documentación láser.179, 1986. 
2. VAÑÓ, E.: "Interacción de las partículas y de los fotones con la materia". Instituto de Estudios Nucleares, CIEMAT. 2000.

*3. GROSS, A.J.; HERRMANN, T.R.: "History of laser". World J. Urol., 25: 217, 2007.

4. EINSTEIN, A.: "Zur Quantentheorie der Strahlung". Phys. Z., 18: 121, 1917.

5. SCHAWLOW, A.L.; TOWNES, C.H.: "Infrared and optical maser". Phys. Rev., 112: 1940, 1959.

6. MAIMAN, T.H.: "Stimulate Optical Radiation in Ruby". Nature, 187: 493, 1960.

7. PARSONS, R.L.; CAMPBELL, J.L.; THOMLEY, M.W.: "Carcinoma of penis treated by the ruby laser". J. Urol., 100: 38, 1968.

8. MULVANY, W.P.; BECK, C.W.: "The laser bearm in urology". J. Urol., 99: 112, 1968.

9. DRETLER, S.P.; WATSON, G.; PARRISH, J.A. y cols.: "Pulse dye laser fragmentations of ureteral calculi: Initial clinical experience”. J. Urol., 137: 386, 1987.

10. BORAICO, A.A.: "A splendid light". National Geographic, 165: 335, 1984.

*11. VICENTE, J.; FERNÁNDEZ, I.; HERNÁNDEZ, C. y cols.: "Láser en urología". Actas Urol. Esp., 30: 879, 2006.

12. JANSEN, E.D.; VAN LEEUWEN, T.G.; MOTAMEDI, M. y cols.: "Temperature dependence of absorption coefficient of water for midinfrared laser radiations". Laser Surg. Med., 14: 258, 1994.

*13. VASSAR, G.J.; CHAN, K.F.; TEICHMAN, J.M. y cols.: "Holmium: YAG photothermal mechanism". J. Endourol., 13: 181, 1999.

14. MESTER, E.; BACSY, E.; KORENYI, A. y cols.: "Effect of laser on wound healing". Am. J. Surg., 11: 532, 1971

15. ADER, W.R.: "Tissue interactions with noionizing electromagnetic fields". Phys. Rew., 61: 435, 1881.

16. ABSTEN, G.T.: "Physics of light and laser". Obstet. Gynecol. Clin. North Am., 18: 407, 1991.

17. BHATTA, K.M.: "Laser in urology". Laser surg. Med., 16: 312, 1995.

18. FLORATOS, D.L.; DE LA ROSETTE, J.J.: "Lasers in urology”. BJU Int., 84: 204, 1999.

19. STEIN, B.S.; KENDALL, A.R.: "Laser in urology". Urology, 23: 411, 1984

20. LOBIK, L.; RAVID, A.; NISSENKORN, I. y cols.: "Bladder welding in rats using controlled temperature CO2 laser system”. J. Urol., 161: 1662, 1999.

21. VICENTE, J.; SALVADOR, J.; LAGUNA, P. y cols.: "Histological evaluation of superficial bladder tumours treated by Nd: YAG laser and transurethral resection". Eur. Urol., 20: 192, 1991.

22. VICENTE, J.; SALVADOR, J.; CAFFARATTI, J.: "Endosocpic urethroctomy versus urethroctomy plus Nd: YAG laser in the treatment of urethral strictures". Eur. Urol., 18: 166, 1900.

23. HELFMANN, J.; MULLER, G.: "Laser lithotripsy: Porces overview". Med. Laser Appl., 16: 30, 2001.

24. MARGUET, C.G.; SUNG, J.C.; SPRINGHART, W.P. y cols.: "In vitro comparison of stone retropulsion and fragmentation of frecuency double-double pulse $\mathrm{Nd}$ : YAG laser and the Holmium: YAG laser". J. Urol., 173: 1797, 2005.

25. DUBOSQ, F.; PASQUI, F.; GIRARD, F. y cols.: "Endocopic lithotripsy and the FREDDY laser: Initial experience". J. Endourol., 20: 296, 2006.

*26. MALEK, R.S.; KUNTZMAN, R.S.; BARRETT, D.M.:
"Photoselective potassium-titanyl-phosphate laser vaporization of the benig obstructive porstate: Observations on long term outcomes". J. Urol., 174: 210, 2005.

27. REICH, O.; BACHMANN, A.; SIEBELS, M.: "High power potassium-titayl-phosphate laser vaporzations of the prostate in 66 high risk patients". J. Urol., 173: 158, 2005.

28. BACHMANN, A.; REICH, O.; WYLER, S. y cols.: "The $80 \mathrm{~W}$ potassium-titayl-phosphate laser vaporization of the prostate. Techinque and 6 moth follow-up after 70 procedures". Urology, 43: 1267, 2004.

29. TUREK, P.J.; MALLOY, T.R.; CENDRON, M, y cols.: "Ktp 352 laser ablation of urethral strictures". Urology, 40: 330, 1992.

30. TEICHMAN, J.M.; VASSAR, G.J.; BISHOFF, J.T. y cols.: "Holmium: YAG lithopripsy fields smaller fragment than lithoclast, pulse dye laser or electrohydruilic lithotripsy". J. Urol., 159: 17, 1998.

*31. SOFER, M.; WATTERSON, J.D.; WOLLIN, T.A. y cols.: "Holmium: YAG lithotripsy for upper tract urinary calculi in 598 patients". J. Urol., 161: 31, 2002.

32. GUILLING, P.J.; CASS, C.B.; CRESSWELL, M.D. y cols.: "The use of the holmium laser in the treatment of benign prostatic hyperplasia". J. Endourol., 10: 459, 1996.

33. GIDDENS, J.L.; GRASSO, M.: "Retrograde ureteroscopic endopyelotomy using the holmium: YAG laser". J. Urol., 164: 1509, 2000.

*34. MATSUOKA, K.; INOUE, M.; IIDA, S. y cols.: "Endoscopic antegrade laser incision in the treatment of urethral stricture". Urology, 60: 968, 2002.

35. LEE, H.; KANG, H.W.; TEICHMAN, J.M. y cols.: "Urinary calculus fragmentation during Ho: YAG and Erb: YAG lithotripsy". Laser Surg. Med., 38: 39, 2006.

36. JONLER, M.; LUND, L.; BISBALLE, S.: "Holmium: YAG laser vaporization of recurrent papillary tumors of the bladder under local anesthesia". BJU Int., 94: 322, 2004.

37. KOENING, F.; McGOVERN, F.J.; ALTHAUSEN, A.F. y cols.: "Laser induced autofluorescence diagnosis of bladder cancer". J. Urol., 165: 1597, 1996.

38. TEICHMAN, J.M.; CHAN, K.F.; CECCONI, P.P. y cols.: "Erbium: YAG versus Holmiun: YAG lithotripsy". J. Urol., 165: 876, 2001.

39. VARKARAKIS, I.M.; INAGAKI, T.; ALLAFA, M.E. y cols.: "Erbium vs Holmium laser incision of urethra and bladder neck". Proceedings of SPIE, vol 5686: págs. 171-175. 2005.

40. CHANEY, C.A.; YANG, Y.; FRIED, N.M.: “Assembly and testing of Germanium/silica Optical fibers for flexible endoscopic delivery of Erbium: YAG laser radiation”. Proceedings of SPIE, vol 5317. págs. 1-8. 2004.

41. DEVOS, D.; CREACH, C.; LAUREAU, E. y cols.: "Thulium laser evoked potentials. Normative values for the arms and legs". Neurophysiol. Clin., 30: 313, 2000.

42. EL-SHERIF, A.F.; KING, T.A.: "Soft and hard tissue ablation with short-pulse high peak power and continuous thulium-silica fiber lasers". Laser Med. Sci., 18: 139, 2003.

43. FRIED, N.M.: "High power laser vaporization of the canine prostate using a $110 \mathrm{~W}$ Thulium fiber laser at 1.91 microns". Laser Surg. Med. 36: 52, 2005.

44. FRIED, N.M.; MURRAY, K.E.: "High power thulium fiber laser ablation of urinary tissues at 1.94 microns". J. Endourol., 19: 25, 2005. 